\title{
INTERAÇÃO DOS FUNGOS MICORRÍZICOS ARBUSCULARES Glomus etunicatum E Gigaspora margarita E O NEMATÓIDE DAS GALHAS Meloidogyne javanica EM TOMATEIRO
}

\author{
ELIS T. COFCEWICZ ${ }^{1}$, CARLOS A.B. MEDEIROS ${ }^{1}$, REGINA M.D.G.CARNEIRO ${ }^{2}$ \& CARLOS R. PIEROBOM $^{3}$ \\ 'Embrapa Clima Temperado, Cx. Postal 403, 96001-970, Pelotas, RS, e-mail: medeiros@cpact.embrapa.br; ${ }^{2}$ Embrapa \\ Recursos Genéticos e Biotecnologia, Cx. Postal 0232, 70770-900, Brasília, DF; ${ }^{3}$ Departamento de Fitopatologia da \\ Universidade Federal de Pelotas, UFPEL, 96001-970, Pelotas, RS
}

(Aceito para publicação em 02/01/2001)

Autor para correspondência: Carlos A.B. Medeiros

COFCEWICZ, E.T., MEDEIROS, C.A.B., CARNEIRO, R.M.D.G. \& PIEROBOM, C.R. Interação dos fungos micorrízicos arbusculares Glomus etunicatum e Gigaspora margarita e o nematóide das galhas Meloidogyne javanica em tomateiro. Fitopatologia Brasileira 26:65-70. 2001.

\section{RESUMO}

A produtividade do tomateiro pode ser severamente reduzida pelo parasitismo dos nematóides das galhas, amplamente distribuídos nas áreas de produção hortícola. Em condições de casa de vegetação, avaliou-se o efeito da interação entre duas espécies de fungos micorrízicos arbusculares (MA), Glomus etunicatum e Gigaspora margarita e o nematóide das galhas, Meloidogyne javanica, sobre o crescimento e nutrição do tomateiro (Lycopersicon esculentum) cv. Floradade. A infecção por M. javanica reduziu a massa de matéria seca da parte aérea das plantas inoculadas. A redução foi menos acentuada em plantas colonizadas com $G$. etunicatum (12\%), comparadas às plantas colonizadas com G. margarita (32\%) e testemunha (24\%). Plantas colonizadas com G. etunicatum apresentaram maior massa de matéria seca e número de frutos comparadas aos demais tratamentos. A produção de frutos foi menos afetada pelo nematóide nas plantas inoculadas com G. etunicatum. A concentração e o conteúdo de $\mathrm{P}$ na parte aérea não foram alterados pelo nematóide nas plantas colonizadas por G. etunicatum, mas foram reduzidos nas plantas colonizadas com G. margarita e testemunhas. O número de ovos e juvenis de segundo estádio de $M$. javanica foi maior nas plantas colonizadas por fungos MA, comparadas às não micorrizadas. A maior tolerância observada nas plantas colonizadas com G. etunicatum ao nematóide das galhas, possivelmente está associada à maior estabilidade na absorção de $\mathrm{P}$.

Palavras-chave: endomicorrizas, fitonematóides, nutrição mineral, fósforo.

\section{ABSTRACT \\ Interaction of arbuscular mycorrhizal fungi Glomus etunicatum and Gigaspora margarita and root-knot nematode Meloidogyne javanica in tomato}

Tomato production may be dramatically reduced by root-knot nematodes, widely spread on horticultural areas. The interaction of arbuscular mycorrhizal fungi Glomus etunicatum and Gigaspora margarita and root-knot nematode, Meloidogyne javanica and their effects on growth and mineral nutrition of tomato (Lycopersicon esculentum) was studied under greenhouse conditions. Shoot dry matter yield was reduced by a nematode infection. The reduction was less pronounced in plants colonized with G. etunicatum (12\%) than in plants colonized with G. margarita (32\%) and nonmycorrhizal plants (24\%). Plants colonized with
G. etunicatum had the highest fruit number and fruit dry weight. Fruit production was less affected by the nematode in plants colonized with G. etunicatum. Shoot P concentration and content were not affected by $M$. javanica in plants colonized with G. etunicatum, but were reduced in plants colonized with G. margarita and nonmycorrhizal plants. Mycorrhizal plants had a higher number of eggs and juveniles of second stage $\left(\mathrm{J}_{2}\right)$ of $M$. javanica than non-mycorrhizal plants. The higher tolerance of plants colonized with G. etunicatum to M. javanica appeared to be associated with $\mathrm{P}$ nutrition

\section{INTRODUÇÃO}

Variabilidade tem sido observada na interação de fungos micorrízicos arbusculares (MA) e fitonematóides, indicando que a associação hospedeiro-nematóide-fungo MA é bastante específica e que alterações em um dos componentes pode levar à diversidade de resultados. Em geral, os dados existentes indicam que as reduções de crescimento, consequiência da infecção por nematóides, têm sido menores em plantas colonizadas por fungos MA comparadas às plantas 
não micorrizadas. Em pessegueiro, a colonização com fungos MA reduziu o estresse causado pelo nematóide Meloidogyne incognita (Kofold \&White) Chitwood, proporcionando plantas mais desenvolvidas, em comparação às inoculadas unicamente com o patógeno (Strobel et al., 1982). Contrariamente, em videira, observou-se que a presença do nematóide Meloidogyne arenaria (Neal) Chitwood suprimiu completamente o efeito benéfico do fungo MA Glomus fasciculatum (Thaxter sensu Gerd) Gerdemann \& Trappe sobre o crescimento e nutrição mineral das plantas colonizadas (Atilano et al., 1976).

A colonização por fungos MA tem, igualmente, afetado a reprodução dos nematóides, reduzindo em muitos casos a ovoposição e o número de indivíduos no sistema radicular de plantas infetadas. Por exemplo, a penetração e o desenvolvimento do nematóide Meloidogyne hapla Chitwood foram inibidos em cebola (Allium cepa L.) colonizada por $G$. fasciculatum, verificando-se que a população final de nematóides foi duas vezes maior em plantas não micorrizadas quando comparadas às plantas colonizadas pelos fungos MA (MacGuidwin et al., 1985). Em tomateiro, demonstrou-se o efeito negativo de $G$. fasciculatum sobre o número e tamanho das galhas formadas por Meloidogyne javanica (Treub) Chitwood e M. incognita (Bagyaraj et al., 1979). Embora um expressivo número de trabalhos evidenciem o efeito negativo de fungos micorrízicos sobre a reprodução de nematóides, a variabilidade existente entre os dados não permite conclusões definitivas.

$\mathrm{O}$ efeito dos fungos MA sobre a reprodução de nematóides, tem sido apontado como dependente de um elevado percentual de colonização da raiz por esses organismos. Saleh \& Sikora (1984) observaram que somente onde a colonização radicular foi maior que $54 \%$ obteve-se efeito sobre a reprodução de $M$. incognita. Da mesma forma, temse observado que o nível da população do nematóide afeta negativamente a colonização e reprodução de fungos micorrízicos. Redução na colonização radicular e na reprodução de fungos MA causadas por Pratylenchus vulnus Allen \& Jensen, com possíveis efeitos sobre a absorção de fósforo e outros nutrientes, foram observadas por Pinochet et al. (1998).

Objetivou-se com este trabalho, avaliar o potencial de duas espécies de fungos MA no controle biológico do nematóide das galhas (M. javanica) em tomateiro.

\section{MATERIAL E MÉTODOS}

O experimento foi conduzido em condições de casa de vegetação, durante os meses de novembro a março, com temperatura variando de 20 a $38{ }^{\circ} \mathrm{C} \pm 1{ }^{\circ} \mathrm{C}$. Sementes de tomateiro cv. Floradade, desinfestadas superficialmente com $\mathrm{NaOCl} 1,0 \%$ por $45 \mathrm{~min}$, foram pré-germinadas e transplantadas para potes plásticos com capacidade seis litros, contendo substrato (solo e areia 1:1), acrescido de $10 \mathrm{~g}$ de calcário/kg de substrato, autoclavado $\left(120^{\circ} \mathrm{C}, 0.15 \mathrm{Mpa}, 120\right.$ $\min )$.
Os tratamentos, com e sem M. javanica, presença e ausência dos fungos MA, Glomus etunicatum Becker \& Gerdemann e Gigaspora margarita Becker \& Hall, foram distribuídos em esquema fatorial, com seis repetições, em blocos inteiramente casualizados. O inóculo utilizado constou de solo contendo esporos, hifas e raízes colonizadas, colocado na porção central do pote, a cerca de $2 \mathrm{~cm}$ de profundidade. Utilizaram-se 10 e $25 \mathrm{~g}$ de inóculo, respectivamente, para $G$. etunicatum e $G$. margarita, contendo a mesma quantidade de esporos, aproximadamente 1.700. Uniformizou-se a microbiota em todos os tratamentos, adicionando-se filtrado preparado a partir do inóculo das duas espécies de fungo MA. A inoculação $\operatorname{com} M$. javanica foi obtida pelo método Hussey e Barker (1973), modificado por Bonetti \& Ferraz (1981), distribuindo-se $10 \mathrm{ml}$ de uma suspensão contendo cerca de 6.000 ovos por pote. As plantas foram colhidas 123 dias após transplantadas. A parte aérea foi desidratada em estufa a 60 ${ }^{\circ} \mathrm{C}$ por 20 dias e as raízes lavadas e coloridas pelo método de Phillips \& Hayman (1970), sendo em seguida avaliadas quanto a percentagem de colonização micorrízica (Giovanneti \& Mosse, 1980). O número de esporos dos fungos MA foram analisados de acordo com o método de Allen et al. (1979). O índice de galhas foi determinado conforme proposto por Taylor $\&$ Sasser (1978). As avaliações do número de ovos por sistema radicular e do número de juvenis de segundo estádio $\left(\mathrm{J}_{2}\right)$ por 100 cc de solo foram feitas após extração pelos métodos de Hussey e Barker (1973), modificado por Bonetti \& Ferraz (1981), e Jenkins (1964), respectivamente.

A determinação dos teores de macro e micronutrientes na parte aérea das plantas foi realizada pelos métodos de Tedesco et al. (1985) e Walinga et al. (1989), respectivamente.

Os dados obtidos foram submetidos à análise de variância pelo teste $\mathrm{F}$ e as médias dos tratamentos comparadas pelo teste Duncan, ao nível de 5\% de probabilidade. Os dados originais relativos ao número de esporos, número de ovos e juvenis, foram transformados em raiz quadrada de $x+0,5$ e os dados da variável percentagem de colonização para arco seno [raiz (x/k)].

\section{RESULTADOS}

A infecção com $M$. javanica reduziu a massa de matéria seca da parte aérea do tomateiro (Tabela 1). As interações fungos MA x nematóide mostraram que essa redução foi mais acentuada nas plantas colonizadas por $G$. margarita $(32 \%)$ e testemunha $(24 \%)$, comparadas as plantas colonizadas por $G$. etunicatum (12\%). Da mesma forma, a infecção pelo nematóide reduziu o peso e número de frutos (Tabela 1). Plantas colonizadas com G. etunicatum apresentaram maior massa de frutos secos e número de frutos, comparadas as plantas inoculadas com G. margarita e testemunha. Os tratamentos $G$. margarita e testemunha não diferiram em relação a produção de matéria seca da parte aérea, massa e número de frutos.

Plantas inoculadas com fungos MA, na presença ou na ausência de $M$. javanica, apresentaram concentração de 
fósforo superior à testemunha (Tabela 2). A análise da interação fungos MA x nematóide mostrou que a infecção com o nematóide não alterou o conteúdo de $\mathrm{P}$ nas plantas colonizadas com G. etunicatum, mas reduziu nos tratamentos G. margarita e testemunha.

O conteúdo e concentração de $\mathrm{Cu}$ na parte aérea das plantas (Tabela 3), foi maior no tratamento G. etunicatum comparado aos tratamentos G. margarita e testemunha. A concentração de $\mathrm{Cu}$ não foi afetada pela inoculação com $M$. javanica.

Os tratamentos com fungos micorrízicos e com nematóide interagiram de forma que a infecção com $M$. javanica diminuiu a concentração de Zn na parte aérea das plantas micorrizadas (Tabela 4), não afetando porém a concentração desse nutriente nas plantas não colonizadas. O conteúdo de Zn na parte aérea das plantas, foi reduzido pela infecção com o nematóide.

A espécie G. etunicatum produziu maior número de esporos do que a G. margarita (Tabela 5). Como resultado da interação entre os tratamentos com fungos micorrízicos e

TABELA 1 - Massa da matéria seca da parte aérea, massa de frutos secos e número de frutos, de plantas de tomateiro (Lycopersicon esculentum) com Glomus etunicatum (G.E.), Gigaspora margarita (G.M.) e testemunha (-MA) na presença (+NEM) ou na ausência (-NEM) de Meloidogyne javanica

\begin{tabular}{|c|c|c|c|c|c|c|}
\hline & \multicolumn{2}{|c|}{$\begin{array}{c}\text { Matéria seca* } \\
\text { (g/planta) }\end{array}$} & \multicolumn{2}{|c|}{$\begin{array}{l}\text { Massa frutos* } \\
\text { (g/planta) }\end{array}$} & \multicolumn{2}{|c|}{ Número de frutos* } \\
\hline & +NEM* & -NEM & +NEM & -NEM & + NEM & -NEM \\
\hline G.E. & $31.8 \mathrm{Ba}$ & $36.3 \mathrm{Aa}$ & $4.5 \mathrm{Ba}$ & $11.0 \mathrm{Aa}$ & $1.5 \mathrm{Ba}$ & $4.5 \mathrm{Aa}$ \\
\hline G.M. & $23.1 \mathrm{Bb}$ & 33.9Aab & $1.8 \mathrm{Bb}$ & $5.3 \mathrm{Ab}$ & $0.6 \mathrm{Ba}$ & $1.6 \mathrm{Ab}$ \\
\hline -MA & $25.2 \mathrm{Bb}$ & $33.0 \mathrm{Ab}$ & $1.9 \mathrm{Bb}$ & $5.3 \mathrm{Ab}$ & $0.8 \mathrm{Ba}$ & $2.6 \mathrm{Ab}$ \\
\hline
\end{tabular}

* Médias seguidas das mesmas letras maiúsculas nas linhas, e minúsculas nas colunas, não diferem estatisticamente pelo teste de Duncan $(\mathrm{P}<0,05)$

TABELA 2 - Concentração e conteúdo de fósforo na parte aérea de plantas de tomateiro (Lycopersicon esculentum) com Glomus etunicatum (G.E.), Gigaspora margarita (G.M.) e testemunha (-MA) na presença (+NEM) ou na ausência (-NEM) de Meloidogyne javanica

\begin{tabular}{|c|c|c|c|c|}
\hline & \multicolumn{2}{|c|}{$\begin{array}{c}\text { Concentração de } \mathrm{P}^{*} \\
(\mathrm{mg} / \mathrm{g})\end{array}$} & \multicolumn{2}{|c|}{$\begin{array}{l}\text { Conteúdo de } \mathrm{P}^{*} \\
\text { (mg/planta) }\end{array}$} \\
\hline & +NEM* & -NEM & +NEM & -NEM \\
\hline G.E. & $1,37 \mathrm{Aa}$ & $1,43 \mathrm{Aa}$ & $43,5 \mathrm{Aa}$ & $51,9 \mathrm{Aa}$ \\
\hline G.M. & $1,12 \mathrm{Bb}$ & $1,43 \mathrm{Aa}$ & $25,8 \mathrm{Bb}$ & $48,4 \mathrm{Aa}$ \\
\hline -MA & $0,62 \mathrm{Bc}$ & $0,93 \mathrm{Ab}$ & $15,6 \mathrm{Bc}$ & $30,7 \mathrm{Ab}$ \\
\hline
\end{tabular}

* Médias seguidas das mesmas letras maiúsculas nas linhas, e minúsculas nas colunas, não diferem estatisticamente pelo teste de Duncan $(\mathrm{P}<0,05)$
TABELA 3 - Concentração e conteúdo de cobre na parte aérea de plantas de tomateiro (Lycopersicon esculentum) com Glomus etunicatum (G.E.), Gigaspora margarita (G.M.) e testemunha (-MA) na presença (+NEM) ou na ausência (-NEM) de Meloidogyne javanica

\begin{tabular}{lcclll}
\hline \hline & \multicolumn{2}{c}{$\begin{array}{c}\text { Concentração de } \mathbf{C u} \\
(\boldsymbol{\mu g} / \mathbf{g})\end{array}$} & & \multicolumn{2}{c}{$\begin{array}{c}\text { Conteúdo de } \mathbf{C u} \\
(\mathbf{m g} / \mathbf{p l a n t a})\end{array}$} \\
\cline { 2 - 3 } \cline { 5 - 6 } \cline { 5 - 6 } & $+\mathbf{N E M *}$ & -NEM & & $+\mathbf{N E M}$ & -NEM \\
\hline G.E. & $8,83 \mathrm{Aa}$ & $8,83 \mathrm{Aa}$ & & $0,28 \mathrm{Ba}$ & $0,31 \mathrm{Aa}$ \\
G.M. & $7,58 \mathrm{Ab}$ & $7,75 \mathrm{Ab}$ & & $0,19 \mathrm{Bb}$ & $0,26 \mathrm{Ab}$ \\
-MA & $7,25 \mathrm{Ab}$ & $6,91 \mathrm{Ab}$ & & $0,18 \mathrm{Bb}$ & $0,23 \mathrm{Ac}$ \\
\hline
\end{tabular}

* Médias seguidas das mesmas letras maiúsculas nas linhas, e minúsculas nas colunas, não diferem estatisticamente pelo teste de Duncan $(\mathrm{P}<0,05)$

TABELA 4 - Concentração e conteúdo de zinco na parte aérea de plantas de tomateiro (Lycopersicon esculentum) com Glomus etunicatum (G.E.), Gigaspora margarita (G.M.) e testemunha (-MA) na presença (+NEM) ou na ausência (-NEM) de Meloidogyne javanica

\begin{tabular}{|c|c|c|c|c|}
\hline & \multicolumn{2}{|c|}{$\begin{array}{c}\text { Concentração de } \mathrm{Zn} \text { * } \\
(\mu \mathrm{g} / \mathrm{g})\end{array}$} & \multicolumn{2}{|c|}{$\begin{array}{c}\text { Conteúdo total de } \mathrm{Zn} * \\
\text { (mg/planta) }\end{array}$} \\
\hline & +NEM* & -NEM & +NEM & -NEM \\
\hline G.E. & $46,2 \mathrm{Bc}$ & $51,7 \mathrm{Ab}$ & $1,48 \mathrm{Bab}$ & $1,83 \mathrm{Aa}$ \\
\hline G.M. & $51,6 \mathrm{Bb}$ & 58,7Aa & $1,31 \mathrm{Bb}$ & 1,99Aa \\
\hline -MA & $62,3 \mathrm{Aa}$ & $61,0 \mathrm{Aa}$ & $1,57 \mathrm{Ba}$ & $2,06 \mathrm{Aa}$ \\
\hline
\end{tabular}

* Médias seguidas das mesmas letras maiúsculas nas linhas, e minúsculas nas colunas, não diferem estatisticamente pelo teste de Duncan $(\mathrm{P}<0,05)$

nematóide, observou-se que a infecção com M.javanica afetou de forma distinta a produção de esporos das duas espécies de fungos MA, com favorecimento a $G$. margarita. Na interação entre os fatores fungos micorrízicos e nematóide, verificouse que a infecção pelo nematóide diminuiu a percentagem de colonização por G. margarita, mas não alterou a colonização por G. etunicatum (Tabela 5).

Plantas micorrizadas apresentaram, no geral, maior número de galhas, consequentemente maior número de ovos e de juvenis de M. javanica que a testemunha (Tabela 6). Nos tratamentos com G. margarita o número de ovos produzidos foi superior ao tratamento G. etunicatum.

\section{DISCUSSÃO}

A concentração de $\mathrm{P}$ na parte aérea, foi inferior ao nível crítico para tomateiro (aproximadamente $2,5 \mathrm{mg} \mathrm{g}^{1}$, Lorenz \& Tyler, 1983). Essa baixa concentração resultou do uso de substrato com reduzido teor desse nutriente para estimular a micorrização. Possivelmente, tenha sido este um 


\section{E.T. Cofcewicz et al.}

TABELA 5 - Número de esporos de Glomus etunicatum (G.E.) e de Gigaspora margarita (G.M.) 100 $\mathrm{g}^{-1}$ de solo e colonização micorrízica de plantas de tomateiro (Lycopersicon esculentum) cv. Floradade na presença (+NEM) ou na ausência de Meloidogyne javanica (- NEM)

\begin{tabular}{ccccccc}
\hline \hline & \multicolumn{2}{c}{$\begin{array}{c}\text { Número de esporos* } \\
\mathbf{1 0 0 g} / \text { solo }\end{array}$} & & \multicolumn{2}{c}{$\begin{array}{c}\text { Colonização micorrízica* } \\
\text { (\%) }\end{array}$} \\
\cline { 2 - 3 } \cline { 5 - 6 } & + +NEM* & -NEM & & + +NEM & -NEM \\
\hline G.E. & $380 \mathrm{Aa}$ & $452 \mathrm{Aa}$ & & $68 \mathrm{Aa}$ & $71 \mathrm{Ab}$ \\
G.M. & $59 \mathrm{Ab}$ & $28 \mathrm{Bb}$ & & $69 \mathrm{Ba}$ & $78 \mathrm{Aa}$ \\
\hline
\end{tabular}

* Médias seguidas das mesmas letras maiúsculas nas linhas, e minúsculas nas colunas, não diferem estatisticamente pelo teste de Duncan $(\mathrm{P}<0,05)$

TABELA 6 - Índice de galhas, número de ovos e de juvenis de Meloidogyne javanica em plantas de tomateiro (Lycopersicon esculentum) cv. Floradade com Glomus etunicatum (G.E.), Gigaspora margarita (G.M.) e Testemunha (MA)

\begin{tabular}{llcc}
\hline \hline & $\begin{array}{c}\text { Índice de } \\
\text { galhas* }^{*}\end{array}$ & $\begin{array}{c}\text { Número de } \\
\text { ovos* }^{*}\end{array}$ & $\begin{array}{c}\text { Número de } \\
\text { juvenis }\left(\mathbf{J}_{2}\right)^{*}\end{array}$ \\
\hline G. E. & $4.6 \mathrm{a}$ & $162 \mathrm{~b}$ & $33 \mathrm{a}$ \\
G.M. & $4.4 \mathrm{ab}$ & $353 \mathrm{a}$ & $22 \mathrm{a}$ \\
-MA & $4.0 \mathrm{~b}$ & $68 \mathrm{c}$ & $9 \mathrm{~b}$ \\
\hline
\end{tabular}

* Médias seguidas das mesmas letras maiúsculas nas linhas, e minúsculas nas colunas, não diferem estatisticamente pelo teste de Duncan $(\mathrm{P}<0,05)$

dos fatores limitantes do crescimento das plantas. O decréscimo na massa de matéria seca da parte aérea e frutos, decorrente do ataque de nematóides pode estar associado à redução dos níveis de $\mathrm{P}$, verificado nas plantas infetadas. A formação de células gigantes, como resultado da infecção pelo nematóide, provoca uma interrupção e desorganização do sistema vascular. Consequentemente, há uma diminuição na absorção e no transporte de água e nutrientes, o que diretamente influencia a produção e qualidade dos frutos. A redução de matéria seca dos frutos e da parte aérea, ocorrida em função da infecção pelo nematóide, foi menor no tratamento $G$. etunicatum. A maior tolerância das plantas colonizadas com G. etunicatum ao nematóide pode ser atribuída, ao menos parcialmente, ao aumento na absorção do $\mathrm{P}$, proporcionado pela associação fungo-hospedeiro. Segundo Smith (1987), o aumento da tolerância a fitonematóides verificado em plantas de tomateiro micorrizadas, resulta de um aumento na absorção de P. Os dados sugerem, no entanto, que o P não foi o único fator envolvido na diferença de produção de matéria seca observada entre os tratamentos. A concentração de $\mathrm{P}$ nas plantas inoculadas com G. margarita foi superior à da testemunha. Entretanto, a produção de matéria seca da parte aérea e frutos foi semelhante nesses dois tratamentos.

Cobre e Zinco, são elementos que por apresentarem lenta difusão no solo, assim como o P, normalmente têm a absorção aumentada pela colonização micorrízica (Pinochet et al., 1998). Plantas colonizadas por G. etunicatum apresentaram maior concentração e conteúdo de $\mathrm{Cu}$, sugerindo que esse nutriente também seja responsável pelas diferenças na produção de matéria seca observadas entre os tratamentos. $\mathrm{O} \mathrm{Cu}$ exerce uma influência direta no desenvolvimento do tomateiro, aumentando o número e tamanho dos frutos, e a matéria seca total da planta (Minami \& Haag, 1979). Os maiores conteúdos de $\mathrm{P}$ e $\mathrm{Cu}$ verificados no tratamento $G$. etunicatum podem ser resultantes de uma maior absorção desses nutrientes ou uma maior translocação das raízes para a parte aérea.

$\mathrm{O}$ aumento da tolerância a Meloidogyne tem sido atribuído a maior absorção de $\mathrm{Zn}$, em conseqüência da colonização por fungos micorrízicos, em plantas inoculadas com os dois microrganismos (Smith et al., 1986). A menor concentração de Zn nas plantas micorrizadas, observada neste estudo, diverge da grande maioria dos dados existentes, onde a colonização micorrízica normalmente determina aumento na concentração desse nutriente (Pinochet et al., 1998). Adicionalmente, redução na absorção de $\mathrm{Zn}$ tem sido relatada como resultado da infecção por Meloidogyne (Minton et al., 1985). Os dados obtidos mostram uma diminuição no conteúdo de Zn causada pelo nematóide, no entanto, a concentração desse nutriente foi reduzida pelo patógeno apenas nas plantas micorrizadas, efeito que não se verificou nas plantas não colonizadas pelos fungos, possivelmente pelo menor nível de infecção pelos nematóides verificados nessas plantas.

A produção de esporos por fungos MA é influenciada pela variação do meio, pelo tipo de hospedeiro e pela espécie de fungo utilizada (Hetrick, 1984). A maior produção de esporos por G. etunicatum está relacionada com características intrínsecas à essa espécie, dada a inexistência, entre os tratamentos, de variabilidade associada ao meio ou ao hospedeiro utilizados. Quando as plantas colonizadas por G. etunicatum foram infetadas pelo nematóide, a esporulação diminuiu. Schenck, et al. (1975) verificaram, igualmente, redução no número de esporos dos fungos MA, associada à altas populações de nematóide. A relação inversa observada para G. margarita, com aumento da esporulação pela infecção com o nematóide, embora já tenha sido observada anteriormente (Gavassoni, 1990), é difícil de ser explicada.

As duas espécies de fungos MA testadas não diferiram entre si, quanto a percentagem de colonização, no entanto, diferiram quanto ao grau de eficiência em estimular o crescimento e a absorção de nutrientes. Os dados confirmam observações de Medeiros et al. (1994), segundo as quais, inexiste correlação entre a colonização e o grau de eficiência de fungos MA. A percentagem de raízes colonizadas foi reduzida em plantas infetadas por $M$. javanica. A presença do nematóide provavelmente, interferiu no fluxo de nutrientes entre os simbiontes, diminuindo o desenvolvimento e a eficiência do fungo. 
Interação dos fungos micorrízicos arbusculares Glomus etunicatum e Gigaspora margarita...

O maior número de galhas verificado nas plantas inoculadas com nematóide e fungos MA, em relação aquelas inoculadas somente com o nematóide, deveu-se, provavelmente, ao maior desenvolvimento do sistema radicular das plantas micorrizadas (observação visual), determinando maior disponibilidade de sítios de infecção para o nematóide. Alguns trabalhos relatam efeito supressivo dos fungos MA sobre o número de ovos de nematóides (Strobel, et al., 1982). No presente estudo, a inoculação com os fungos MA, promoveu um incremento no número de ovos e juvenis por grama de raiz. Resultados semelhantes foram relatados por Hussey \& Roncadori (1982), onde plantas de amendoim (Arachis hypogaea L.) inoculadas com $G$. etunicatum ou $G$. margarita, infetadas por $M$. arenaria, apresentaram um número oito vezes maior de ovos de nematóide por grama de raiz, quando comparadas com plantas não colonizadas. Várias razões podem explicar a maior reprodução do nematóide nas plantas colonizadas. Raízes micorrizadas podem atrair um maior número de juvenis infetivos do que as raízes não micorrizadas, pela maior liberação de exsudatos, determinando uma maior eclosão de ovos. A colonização pelos fungos MA pode aumentar o desenvolvimento do sistema radicular, resultando em um maior número de sítios de penetração e maior sobrevivência póspenetração dos juvenis. Por último, fêmeas adultas podem produzir maior número de ovos sobre plantas micorrizadas, normalmente mais vigorosas.

Embora a maior tolerância das plantas colonizadas com G. etunicatum a $M$. javanica, sob o ponto de vista epidemiológico, a maior reprodução do nematóide nas plantas micorrizadas, seria um fator limitante para uso do fungo micorrízico como agente de controle biológico.

\section{REFERÊNCIA BIBLIOGRÁFICAS}

ALLEN, M.F., MOORE JR, T.S., CHRISTENSEN, M. \& STANTON, N. Growth of vesicular-arbuscularmycorrhizal and nonmycorrhizal Bouteloua gracilis in a defined medium. Mycologia 71:666-669. 1979.

ATILANO, R.A., RICH, J.R., FERRIS, H. \& MENGE, J.A. Effect of Meloidogyne arenaria on endomycorrhizal grape (Vitis vinifera) rootings. Journal of Nematology 8:278. 1976.

BAGYARAJ, D.J., MANJUNATH, A., REDDY, D.D.R. Interaction of vesicular arbuscular mycorrhiza with root knot nematodes in tomato. Plant and Soil 81:397-403. 1979.

BONETTI, J.I.S. \& FERRAZ, S. Modificação do método de Hussey \& Barker para extração de ovos de Meloidogyne exigua em raízes de cafeeiro. Fitopatologia Brasileira 6:553. 1981.

GAVASSONI, W.L. Interação entre Glomus etunicatum ( Becker \& Gerdemann), Meloidogyne incognita (Kofoid \& White, 1919) Chitwood, 1949 e níveis de fósforo em pimenta-do-reino (Piper nigrum L.) (Tese de Mestrado) Viçosa. Universidade Federal de Viçosa, 1990.
GIOVANNETI, M.\& MOSSE, B. An evaluation of technique for measuring vesicular-arbuscular infection in roots. New Phytology 84:489-500.1980.

HETRICK, B.A.D. Ecology of VA Mycorrhizal Fungi. In: POWELL, C.L. \& BAGYARAJ, D.J. (Eds.) VA mycorrhiza. Boca Raton. CRC. Press., 1984. pp.35-56.

HUSSEY, R.S. \& BARKER, K.R. A comparison of methods of collecting inoculo for Meloidogyne spp. including a new technique. Plant Disease Reporter 57:1025-1028. 1973.

HUSSEY, R.S. \& RONCADORI, R.W. Vesicular-arbuscular mycorrhizae may limit nematode activity and improve plant growth. Plant Disease 66:9-14. 1982.

JENKINS, W.R. A rapid centrifugal-flotation technique for separating nematodes from soil. Plant Disease Reporter 48:692. 1964.

LORENZ, O.A. \& TYLER, K.B. Plant tissue analysis of vegetable crops. California. University of California, 1983.

MACGUIDWIN, A.E., BIRD, G.W. \& SAFIR, G.R. Influence of Glomus fasciculatum on Meloidogyne hapla infecting Allium cepa. Journal of Nematology 17:389-395. 1985.

MEDEIROS, C.A.B., CLARK, R.B. \& ELLIS, J.R. Growth and nutrient uptake of sorghum cultivated with vesiculararbuscular mycorrhiza isolates at varying $\mathrm{pH}$. Mycorrhiza,4:185-191. 1994.

MINAMI, K. \& HAAG, H.P. O tomateiro. Campinas: Fundação Cargil, 1979.

MINTON, N.A., PARKER, M.B. \& SUMNER, D.R. Nematode control related to fusarium wilt in soybean and root rot and zinc deficiency in corn. Journal of Nematology 17:314-321. 1985.

PHILLIPS, J.M. \& HAYMAN, D.S. Improved procedures for clearing roots and staining parasitic ans vesiculararbuscular mycorrhizal fungi for rapid assesssment of infection. Transactions of British Mycological Society 55:158-161. 1970.

PINOCHET, J., CAMPRUBÍ, C., CALVET, C. \& FERNÁNDEZ, C. Inducing tolerance to the root-lesion nematode Pratylenchus vulnus by early mycorrhizal inoculation of micropropagated Myrobalan 29 plum rootstock. Journal of American Society of Horticultural Science 123:342-347. 1998.

SALEH, H. \& SIKORA, R.A. Relationship between Glomus fasciculatum root colonization of cotton and its effect on Meloidogyne incognita. Nematologica 30:230-237. 1984.

SCHENCK, N.C., KINLOCH, R.A. \& DICKSON, D.W. Interaction of endomycorrhizal fungi and root-knot nematode on soybean. In: SANDERS, F.E., MOSSE, B. \& TINKER, P.B. (Eds.) Endomycorrhizas. London. Academic Press. 1975. pp.605-617.

SMITH, G.S. Interaction of nematodes with mycorrhizal fungi. In: VEECH, J.A. \& DICKSON, D.W. (Eds.) Vistas on Nematology. Hyattsville. Society of Nematologists. 1987. pp.292-300.

SMITH, G.S., RONCADORI, R.W. \& HUSSEY, R.S. 


\section{E.T. Cofcewicz et al.}

Interaction of endomycorrhizal fungi, superphosphate, and Meloidogyne incognita on microplot and field studies. Journal of Nematology 18:208-216. 1986.

STROBEL, N.E., HUSSEY, R.S. \& RONCADORI, R.W. Interactions of vesicular-arbuscular mycorrhizal fungi, Meloidogyne incognita, and soil fertility on peach. Phytopathology 72:690-694. 1982.

TAYLOR, A.L. \& SASSER, J.N. Biology, identification and control of root-knot nematodes (Meloidogyne spp.). Raleigh, M.C. North Caroline State University. 1978. TEDESCO, M.J., VOLKWEISS, S.J. \& BOHNEN, H. Análises de solos, plantas e outros materiais. Porto Alegre: UFRGS. Boletim Técnico, 5. 1985.

WALINGA, I., VAN VARK, W., HOUBA, V.J.G. \& VAN DER LEE, J.J. Plant Analysis Precedures. Wageningen, The Netherlands: Agricultural University. 1989. 